\title{
Tuning the Relaxation of Imprinted Polymer Films with Polymer-Grafted Nanoparticles
}

Sonal Bhadauriya ${ }^{1}$, Xiaoteng Wang ${ }^{1}$, Dharmaraj Raghavan ${ }^{2}$, Michael R. Bockstaller ${ }^{3}$, Christopher M. Stafford $^{4}$, Jack F. Douglas*4, Alamgir Karim*1

1. Department of Polymer Engineering, The University of Akron, Ohio, 44325 USA

2. Department of Chemistry, Howard University, Washington, District of Columbia 20059, USA

${ }^{3 .}$ Department of Materials Science and Engineering, Carnegie Mellon University, Pittsburgh, Pennsylvania 15213, USA

4. Materials Science and Engineering Division, National Institute of Standards and Technology, Gaithersburg, Maryland 20899, USA

* Corresponding author: Alamgir Karim, akarim3@central.uh.edu; Jack F. Douglas, jack.douglas@nist.gov

Tuning the stability of imprinted patterns in polymer thin films presents an important problem from both fundamental and application standpoint. Surfacial patterns are utilized in many applications such as lithography, nanodevices and biosensors where a decay in the stability of these structures can render them unusable. The stability of imprinted nanostructures in homopolymer thin films has been extensively studied, using both ex-situ ${ }^{[1]}$ and in-situ ${ }^{[2]}$ procedures. For low molecular mass unentangled polymer films, the decay of imprinted nanostructures, termed "slumping", is governed by Laplace pressure induced viscous flow ${ }^{[1,3]}$ so that residual stress effects arising in imprinted high molecular mass films are not a complication ${ }^{[1],[2]}$. Previous work has shown that molecular additives can efficiently stabilize nanoimprinted patterns, ${ }^{[4]}$ and it is natural to investigate whether PGNPs can serve this purpose given the significant influence nanoparticles have on the properties of nanocomposite materials ${ }^{[5]}$. Polymer nanocomposites offer immense possibilities for creating new functional materials through the control of the size and organization of the PGNP within the polymer matrix ${ }^{[6]}$. The effect of PGNPs in imparting structural stability is essentially unexplored.

We investigate the slumping effect in imprinted poly(methyl methacrylate) (PMMA) $\left(\mathrm{M}_{\mathrm{n}}=3.1 \mathrm{~kg} / \mathrm{mol}\right.$, where $\mathrm{M}_{\mathrm{n}}$ is the number average molecular mass) thin films containing PMMA $\left(\mathrm{M}_{\mathrm{n}} \approx 15 \mathrm{~kg} / \mathrm{mol}\right)$ grafted Titania $\left(\mathrm{TiO}_{2} \mathrm{PGNP}\right)$ and PMMA $\left(\mathrm{M}_{\mathrm{n}} \approx 19.4 \mathrm{~kg} / \mathrm{mol}\right)$ grafted Silica $\left(\mathrm{SiO}_{2} \mathrm{PGNP}\right)$ particles. The grafted PMMA chains in this study have a molecular mass that is higher than the matrix molecular mass, corresponding to the so-called "wet brush" regime ${ }^{[7]}$ where the grafted chains are expected to be swollen by the polymer matrix chains. The topography of imprinted samples was characterized in terms of pattern height $(H)$ using ex-situ atomic force microscopy $(A F M)$ after the samples were subjected to different annealing temperatures $(T)$ and exposure time $(t)$ above the ellipsometry-estimated bulk $T_{g}$ value of the PMMA matrix, $T_{g}$, bulk $\approx 97.24( \pm 0.23){ }^{\circ} \mathrm{C}$. Analysis of topographical height data from AFM gives an indirect estimate of the structural stability of the patterns and paves the use of this technique in determining the relaxation behaviour of polymer and their composites.

Addition of PGNP significantly improved the thermal stability above $T_{g}$ and these additives act as stabilizing agents for the patterned nanostructures as can be seen in Figure 1. As the PGNP loading is increased, the patterned nanostructure exhibited a longer relaxation time, indicative of an improved pattern stability. Notably, the stabilization of the imprinted nanostructures relates to the slowing down of 
film relaxation dynamics, where we find an entropy-enthalpy compensation (EEC) effect in the thermally activated free energy parameters governing the "slumping" of the nanoimprinted patterns. EEC is encountered in the dynamics of many condensed materials ${ }^{[8]}$ and corresponds to a linear dependence between the Arrhenius prefactor, $\ln \left(\tau_{0}\right)$, which scales linearly with the entropy of activation, and the activation enthalpy, $E_{a}$. EEC effect was observed in the relaxation dynamics of imprinted composite films and seems to be independent of the dispersion of the PGNP as it is robustly observed for both the systems investigated, clustered ( $\left.\mathrm{TiO}_{2} \mathrm{PGNP}\right)$ and well-dispersed $\left(\mathrm{SiO}_{2} \mathrm{PGNP}\right)$.

In summary, the addition of PGNP to thin PMMA films leads to substantial stabilization of nanoimprinted patterns and provide a simple strategy to control high temperature nano-structural integrity in imprinted polymer films. The relaxation dynamics of the imprinted patterns show a clear EEC effect in the activation free energy parameters that govern the pattern relaxation process, just as in recent experiments on relaxation of wrinkled thin polymer films of variable thickness ${ }^{[9]}$. Our results suggest a common origin of EEC relaxation in imprinted thin film polymer nanocomposites. This study paves the way for using PGNP as stabilizing agents for imprinted structures in various applications and provides fundamental insight into how PGNP influence the dynamics of nanocomposite materials ${ }^{[10]}$.
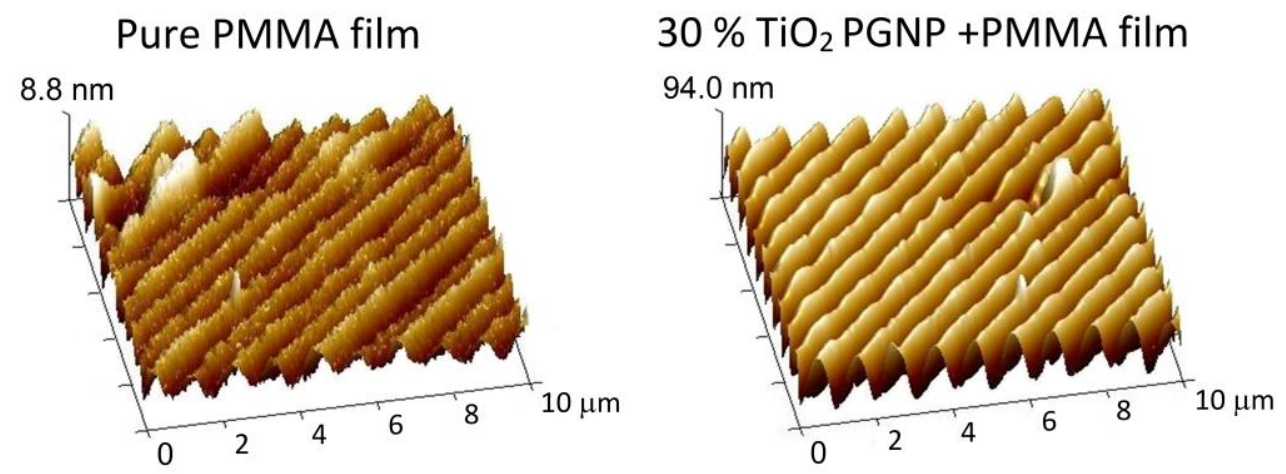

Figure 1. Atomic force microscopy (AFM) height image showing the effect of adding $\mathrm{TiO}_{2} \mathrm{PGNP}$ to a PMMA film, after annealing at $115^{\circ} \mathrm{C}$ for 90 min where the composite film displays a $94 \%$ higher pattern height retention. $\mathrm{TiO}_{2}$ PGNP concentration in the composite film was $30 \%$ by mass relative to the PMMA matrix.

\section{References:}

[1] C. L. Alvine, et. al., J. Polym. Sci. Part B Polym. Phys. 2009, 47, 2591.

[2] Y. Ding, et.al., Adv. Mater. 2007, 19, 1377.

[3] K. J. Alvine, et.al., Soft Matter 2009, 5, 2913.

[4] T. Tanaka, et.al., Jpn. J. Appl. Phys. 1994, 33, L1803.

[5] S. Cheng, et.al., ACS Nano 2017, 11, 752.

[6] J. Kao, et.al., Chem. Soc. Rev. 2013, 42, 2473.

[7] L. S. Schadler, et.al., MRS Bull. 2007, 32, 335.

[8] C. E. Waring, P. Becher, J. Chem. Phys. 1947, 15, 488.

[9] J. Y. Chung, J. F. Douglas, C. M. Stafford, J. Chem. Phys. 2017, 147, 154902 (1-8).

[10] The authors would like to acknowledge DOE (BES) award DE-SC0018854 for funding of this project. 